\title{
Spinning Dust Emission from Wobbling Grains: Important Physical Effects and Implications
}

\author{
Thiem Hoang ${ }^{1}$ and A. Lazarian ${ }^{2}$ \\ ${ }^{1}$ Canadian Institute for Theoretical Astrophysics, University of Toronto, 60 St. George Street, Toronto, \\ ON, Canada M5S $3 \mathrm{H} 8$ \\ ${ }^{2}$ Department of Astronomy, University of Wisconsin-Madison, Madison, WI 53705, USA
}

Correspondence should be addressed to Thiem Hoang, hoang@cita.utoronto.ca

Received 14 August 2012; Accepted 24 October 2012

Academic Editor: Laurent Verstraete

Copyright (C) 2012 T. Hoang and A. Lazarian. This is an open access article distributed under the Creative Commons Attribution License, which permits unrestricted use, distribution, and reproduction in any medium, provided the original work is properly cited.

We review the major progress on the modeling of electric dipole emission from rapidly spinning tiny dust grains, including polycyclic aromatic hydrocarbons (PAHs). We begin by summarizing the original model of spinning dust proposed by Draine and Lazarian and recent theoretical results improving the Draine and Lazarian model. The paper is focused on important physical effects that were disregarded in earlier studies for the sake of simplicity and recently accounted for by us, including grain wobbling due to internal relaxation, impulsive excitation by single-ion collisions, the triaxiality of grain shape, charge fluctuations, and the turbulent nature of astrophysical environments. Implications of the spinning dust for constraining the physical properties of ultrasmall dust grains and environmental conditions are discussed. We discuss the alignment of tiny dust grains and the possibility of polarized spinning dust emission. Suggestions for constraining the alignment of tiny grains and polarization of spinning dust are also discussed.

\section{Introduction}

Diffuse Galactic microwave emission carries important information on the fundamental properties of the interstellar medium, but it also interferes with cosmic microwave background (CMB) experiments (see Bouchet et al. [1] and Tegmark et al. [2]). Precision cosmology with Wilkinson Microwave Anisotropy Probe (WMAP) and Planck satellite requires a good model of the microwave foreground emission to allow for reliable subtraction of Galactic contamination from the $\mathrm{CMB}$ radiation.

The discovery of an anomalous microwave emission (hereafter AME) in the range from $10-100 \mathrm{GHz}$ illustrates well the treacherous nature of dust. Until very recently, it has been thought that there are three major components of the diffuse Galactic foreground: synchrotron emission, freefree radiation from plasma (thermal bremsstrahlung), and thermal emission from dust. In the microwave range, the latter is subdominant, leaving essentially two components. However, it is exactly in this range that an anomalous emission component was reported (Kogut et al. $[3,4]$ ). In the paper by De Oliveira-Costa et al. [5], this emission was nicknamed "Foreground X," which properly reflects its mysterious nature. This component is spatially correlated with $100 \mu \mathrm{m}$ thermal dust emission, but its intensity is much higher than one would expect by directly extrapolating the thermal dust emission spectrum to the microwave range.

An early explanation for AME was proposed by Draine and Lazarian model $[6,7]$ (hereafter DL98 model), where it was identified as electric dipole emission from very small grains (mostly containing polycyclic aromatic hydrocarbons-PAHs) that spin rapidly due to several processes, including gas-grain interactions and dust infrared emission. Although spinning dust emission had been discussed previously (see Erickson [8] and Ferrara and Dettmar [9]), Draine and Lazarian were the first to include the variety of excitation and damping processes that are relevant for very small grains.

While the DL98 model appears to be in general agreement with observations (see $[10,11]$ ), it did not account for 
some important effects, namely, the nonsphericity of grain shapes, the internal relaxation within grain, and the transient spinup due to ion collisions.

This induced more recent work in order to improve the original DL98 model. The recent papers include AliHaïmoud et al. [12], Hoang et al. [13], Ysard and Verstraete [14], Hoang et al. [15], Silsbee et al. [16]. In this paper, we review both the original DL98 model and the ways that it has been improved recently. We focus on the improvement of the dynamics of PAHs and the important physical effects associated with these ultrasmall grains. Recent reviews of the subject include Draine and Lazarian [17], Lazarian and Prunet [18], and Lazarian and Finkbeiner [10].

In Section 2, we briefly present the history of AME and discuss the original DL98 model including their basic assumptions. Section 3 presents our principal results improving the DL98 model from Hoang et al. [13, 15]. From Section 4 to Section 6, we review the grain rotational dynamics and discuss our general approach to calculate power spectrum of spinning dust emission, grain angular momentum distribution, and emissivity for PAHs of arbitrary shapes. In Section 7, we discuss the implications of spinning dust for constraining physical parameters of PAHs as well as environmental conditions. The possibility of polarization of spinning dust and its constraint is discussed in Section 8. A summary of the present paper is given in Section 9.

\section{The Original DL98 Model}

2.1. Anomalous Microwave Emission and PAHs. The emission spectrum of diffuse interstellar dust was mostly obtained by the Infrared Astronomy Satellite (IRAS) and infrared spectrometers on the Cosmic Background Explorer (COBE) and on the Infrared Telescope in Space (IRTS). The emission at short wavelength $(\lambda<50 \mu \mathrm{m})$ arises from transiently heated ultrasmall grains (e.g., PAHs). These grains have such a small heat capacity that the absorption of a single ultraviolet (UV) starlight photon $(\sim 6 \mathrm{eV})$ raises their temperature to $T_{\text {vib }}>$ $200 \mathrm{~K}$. Typically, these grains have less than 300 atoms and can be viewed as large molecules rather than dust particles. They are, however, sufficiently numerous to account for most of the prominent $2175 \AA$ absorption features and for $\sim 35 \%$ of the total starlight absorption (see, e.g., Li and Draine [19]).

The thermal (vibrational) emissivity of these grains is thought to be negligible at low frequency, because they spend most of their time cold and only emit most of their energy when they are hot. These ultrasmall grains (PAHs) are invoked in the DL98 model to account for the anomalous microwave emission (AME) that was measured in observations.

The first detection of anomalous dust-correlated emission by COBE (Kogut et al. [3, 4]) was quickly followed by detections in the data sets from Saskatoon (de Oliveira-Costa et al. [20]), OVRO (Leitch et al. [21]), the $19 \mathrm{GHz}$ survey (De Oliveira-Costa et al. [22]), de Oliveira-Costa et al. ([23]). Initially, AME was identified as thermal bremsstrahlung from ionized gas correlated with dust (Kogut et al. [3]) and was presumably produced by photoionized cloud rims (McCullough et al. [24]). This idea was scrutinized in Draine and Lazarian [6] and criticized on energetic grounds. Poor correlation of $\mathrm{H} \alpha$ with $100 \mu \mathrm{m}$ emission also argued against the free-free explanation (McCullough et al. [24]). These arguments are summarized in [17]. Later, [25] used Wisconsin H-Alpha Mapper (WHAM) survey data and established that the free-free emission "is about an order of magnitude below Foreground $\mathrm{X}$ over the entire range of frequencies and latitudes where it is detected." The authors concluded that the Foreground X cannot be explained as the free-free emission. Additional evidence supporting this conclusion has come from a study at 5,8 , and $10 \mathrm{GHz}$ by Finkbeiner et al. [26] of several dark clouds and HII regions, two of which show a significantly rising spectrum from 5 to $10 \mathrm{GHz}$.

The recent Wilkinson Microwave Anisotropy Probe (WMAP) data were used to claim a lower limit of 5\% for the spinning dust fraction at $23 \mathrm{GHz}$ (Bennett et al. [27]). However, other models of spinning dust are not ruled out by the WMAP data and in fact fit reasonably well. Finkbeiner [11] performed a fit to WMAP data using a CMB template, a free-free template (based on $\mathrm{H} \alpha$-correlated emission plus hot gas emission near the Galactic center), a soft synchrotron template traced by the $408 \mathrm{MHz}$ map, a thermal dust extrapolation (Finkbeiner et al. [28]), and a spinning dust template consisting of dust column density times $T_{d}^{3}$. This fit results in excellent $\chi^{2} / d o f$ values of 1.6, $1.09,1.08,1.05$, and 1.08 at 23, 33, 41, 61, and $94 \mathrm{GHz}$ and a reasonable spectral shape for the average spinning dust spectrum.

This WMAP analysis alone does not rule out the Bennett et al. [27] hypothesis of hard synchrotron emission, but when it is combined with the Green Bank Galactic Plane survey data (Langston et al. [29]) at 8 and $14 \mathrm{GHz}$, spinning dust appears to provide a much better fit than hard synchrotron (Finkbeiner et al. [30]).

Spinning dust emission has recently been reported in a wide range of astrophysical environments, including general ISM (Gold et al. [31, 32] and Collaboration et al. [33]), starforming regions in the nearby galaxy NGC 6946 (Scaife et al. $[34,35]$ ), and Perseus and Ophiuchus clouds (Casassus et al. [36] and Tibbs et al. [37]). Early Planck results have been interpreted as showing a microwave emission excess from the spinning dust in the Magellanic Clouds (Bot et al. [38]; Collaboration et al. [33]).

\subsection{Basic Assumptions}

(i) The smallest PAH particles of a few Angstroms are expected to be planar. The grain size $a$ is defined as the radius of an equivalent sphere of the same mass. PAHs are assumed to be planar, disklike with height $L$ and radius $R$ for $a<a_{2}$, and spherical for $a \geq a_{2}$. The value $a_{2}=6 \AA$ is adopted.

(ii) PAHs usually have electric dipole moment $\boldsymbol{\mu}$ arising from asymmetric polar molecules or substructures (intrinsic dipole moment) and from the asymmetric 
distribution of grain charge. The latter is shown to be less important.

(iii) The grain spins around its symmetry axis $\mathbf{a}_{1}$ with angular momentum $\mathbf{J}$ parallel to $\mathbf{a}_{1}$, and $\mathbf{J}$ is isotropically oriented in space.

(iv) For a fixed angular momentum, the spinning grain emits electric dipole radiation at a unique frequency mode $v$, which is equal to the rotational frequency, that is, $v=\omega / 2 \pi$.

(v) A grain in the gas experiences collisions with neutral atoms and ions, interacts with passing ions (plasmagrain interactions), emits infrared photons following UV absorption, and emits electric dipole radiation. All these processes result in the damping and excitation of grain rotation, that is, they change grain angular momentum $J$ and velocity $\omega$.

(vi) Due to the excitation of various aforementioned processes, the grain angular velocity randomly fluctuates and its distribution can be approximated as the Maxwellian distribution function $f_{\mathrm{Mw}}(\omega)$.

(vii) The total emissivity per $H$ atom of the electric dipole radiation from spinning dust at the frequency $v$ is given by

$$
\frac{j_{v}}{n_{H}}=\frac{1}{4 \pi} \frac{1}{n_{H}} \int_{a_{\min }}^{a_{\max }} d a \frac{d n}{d a} 4 \pi \omega^{2} f_{\mathrm{Mw}}(\omega) 2 \pi\left(\frac{2 \mu_{\perp}^{2} \omega^{4}}{3 c^{3}}\right),
$$

where $n_{H}$ is the density of $H$ nuclei, $\mu_{\perp}$ is the electric dipole moment perpendicular to the rotation axis, and $d n / d a$ is the grain size distribution function with $a$ in the range from $a_{\min }$ to $a_{\max }$.

\section{Improved Model of Spinning Dust Emission}

Ali-Haïmoud et al. [12] revisited the spinning dust model and presented an analytic solution of the Fokker-Planck (FP) equation that describes the rotational excitation of a spherical grain if the discrete nature of impulses from singleion collisions can be neglected.

Hoang et al. [13] (hereafter HDL10) improved the DL98 model by accounting for a number of physical effects. The main modifications in their improved model of spinning dust emission are as follows.

(i) Disk-like grains rotate with their grain symmetry axis $\mathbf{a}_{1}$ that is not perfectly aligned with angular momentum $\mathbf{J}$. The disaligned rotation of $\mathbf{J}$ with $\mathbf{a}_{1}$ causes the wobbling of the grain principal axes with respect to $\mathbf{J}$ due to internal thermal fluctuations.

(ii) The power spectrum of a freely spinning grain is obtained using Fourier transform.

(iii) Distribution function of grain angular momentum, $J$, and velocity, $\omega$, is obtained exactly using the Langevin equation (LE) for the evolution of $\mathbf{J}$ in an inertial coordinate system. (iv) The limiting cases of fast internal relaxation and no internal relaxation are both considered for calculations of the angular momentum distribution and emissivity of spinning dust.

(v) Infrequent collisions of single ions which deposit an angular momentum larger than the grain angular momentum prior to the collision are treated as Poisson-distributed events.

The wobbling disk-like grain has anisotropic rotational damping and excitation. Such an anisotropy can increase the peak emissivity by a factor $\sim 2$ and increases the peak frequency by a factor 1.4-1.8, compared to the results from the DL98 model.

The effects of grain wobbling on electric dipole emission were independently studied in Silsbee et al. [16] using the FP equation approach, but they disregarded the transient spinup by infrequent single-ion collisions and considered two limiting cases of dust grain temperature, $T_{d} \rightarrow 0$ and $T_{d} \rightarrow \infty$.

Further improvements of the DL98 model were performed in Hoang et al. [15], where a couple of additional effects were taken into account:

(i) emission from very small grains of triaxial ellipsoid (irregular) shape with the principal moments of inertia $I_{1} \geq I_{2} \geq I_{3}$,

(ii) effects of the orientation of dipole moment $\boldsymbol{\mu}$ within grain body for different regimes of internal thermal fluctuations,

(iii) effects of compressible turbulence on the spinning dust emission.

The work found that a freely rotating irregular grain with a given angular momentum radiates at multiple frequency modes. The resulting spinning dust spectrum has peak frequency and emissivity increasing with the degree of grain shape irregularity, which is defined by $I_{1}: I_{2}: I_{3}$. Considering the transient heating of grains by UV photons, the study found that the spinning dust emissivity in the case of strong thermal fluctuations is less sensitive to the orientation of $\boldsymbol{\mu}$ than in the case of weak thermal fluctuations. In addition, the emission in a turbulent medium increases by a factor from 1.2-1.4 relative to that in a uniform medium, as sonic Mach number $M_{s}$ increases from 2-7. The latter Mach numbers are relevant to cold phases of the ISM (see Hoang et al. [15] for more details).

\section{Grain Rotational Configuration and Power Spectrum}

A discussion of the basic physical processes involved in spinning dust can be found in the review by Yacine AliHaïmoud, which can be found in the same volume. There, the use of Fokker-Planck equation for describing grain dynamics is discussed. Here, we discuss our numerical approach based on Fourier transform and the Langevin equation, which exhibits a number of advantages to the FP equation when numerical studies of grain dynamics are 
performed and arbitrary shape of PAHs is considered. We summarize a general approach to find the spinning dust emissivity from grains of triaxial ellipsoid shape with $I_{1}>$ $I_{2}>I_{3}$ subject to fast internal relaxation.

4.1. Torque-Free Motion and Internal Relaxation. The dynamics of a triaxial (irregular) grain is more complicated than that of a disk-like grain with $I_{2}=I_{3}$. Indeed, in addition to the precession of the axis of major inertia $\mathbf{a}_{1}$ around $\mathbf{J}$ as in the disk-like grain, the axis $\mathbf{a}_{1}$ wobbles rapidly, resulting in the variation of the angle $\theta$ between $\mathbf{a}_{1}$ and $\mathbf{J}$ (see Figure 1).

To describe the torque-free motion of an irregular grain having a rotational energy $E_{\text {rot }}$, the conserved quantities are taken, including the angular momentum $\mathbf{J}$, and $\mathbf{a}$ dimensionless parameter that characterizes the deviation of the grain rotational energy from its minimum value

$$
q=\frac{2 I_{1} E_{\mathrm{rot}}}{J^{2}}
$$

The orientation of the triaxial grain in the lab system is completely described by three Euler angles $\psi, \phi$, and $\theta$ (see, e.g., Hoang et al. [15]). Following [39], we define the total number of states $s$ in phase space for $q$ ranging from 1 to $q$ as

$$
s \equiv 1-\frac{2}{\pi} \int_{0}^{\psi_{1}} d \psi\left[\frac{I_{3}\left(I_{1}-I_{2} q\right)+I_{1}\left(I_{2}-I_{3}\right) \cos ^{2} \psi}{I_{3}\left(I_{1}-I_{2}\right)+I_{1}\left(I_{2}-I_{3}\right) \cos ^{2} \psi}\right]^{1 / 2},
$$

where

$$
\psi_{1}=\cos ^{-1}\left[\frac{I_{3}\left(I_{2} q-I_{1}\right)}{I_{1}\left(I_{2}-I_{3}\right)}\right]^{1 / 2}
$$

for $q>q_{\mathrm{sp}}$ and $\psi_{1}=\pi / 2$ for $q \leq q_{\mathrm{sp}}$, with $q_{\mathrm{sp}} \equiv I_{1} / I_{2}$ being the separatrix between the two regimes.

The intramolecular vibrational-rotational energy transfer process (IVRET) due to imperfect elasticity occurs on a timescale $10^{-2} \mathrm{~s}$, for a grain of a few angstroms (Purcell [40]), which is shorter than the IR emission time. So, when the vibrational energy decreases due to IR emission, as long as the vibrational-rotational (V-R) energy exchange exists, interactions between vibrational and rotational systems maintain a thermal equilibrium, that is, $T_{\text {rot }} \approx T_{\text {vib. }}$. As a result, the LTE distribution function of rotational energy reads (hereafter VRE regime; see Lazarian and Roberge [41])

$$
f_{\mathrm{VRE}}(s, J) \propto \exp \left(-\frac{E_{\text {rot }}}{k_{B} T_{\text {rot }}}\right) \approx \exp \left(-\frac{E_{\text {rot }}}{k_{B} T_{\text {vib }}}\right) .
$$

Substituting $E_{\text {rot }}$ as a function of $J$ and $q$ from (2) into (5), the distribution function for the rotational energy becomes

$$
f_{\mathrm{VRE}}(s, J)=A \exp \left(-\frac{q(s) J^{2}}{2 I_{1} k_{B} T_{\mathrm{vib}}}\right)
$$

where $A$ is a normalization constant such that $\int_{0}^{1} f_{\mathrm{VRE}}(s, J) d s$ $=1$.

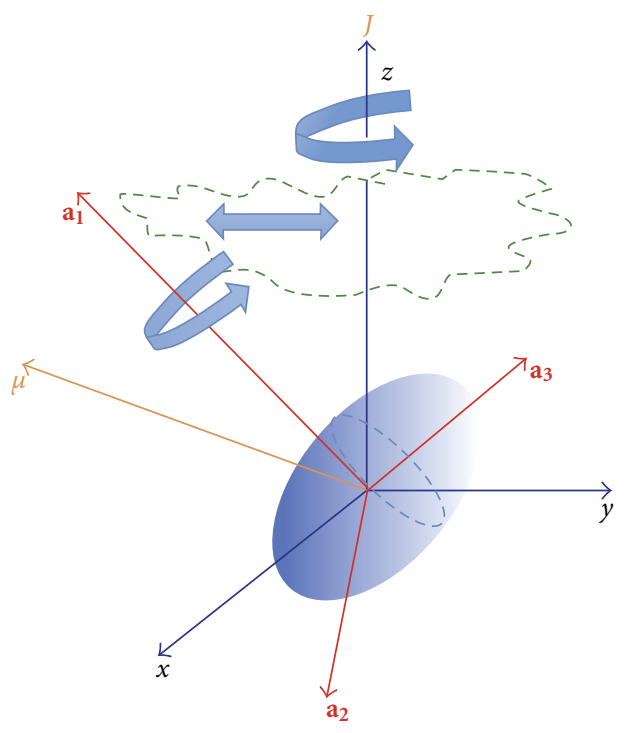

FIGURE 1: Rotational configuration of a triaxial ellipsoid characterized by three principal axes: $\mathbf{a}_{1}, \mathbf{a}_{2}$, and $\mathbf{a}_{3}$ in the inertial coordinate system $x y z$. Grain angular momentum $\mathbf{J}$ is conserved in the absence of external torques and directed along $z$-axis. The torque-free motion of the triaxial grain comprises the rotation around the axis of major inertia $\mathbf{a}_{1}$, the precession of $\mathbf{a}_{1}$ around $\mathbf{J}$, and the wobbling of $\mathbf{a}_{1}$ with respect to $\mathbf{J}$. The dipole moment $\boldsymbol{\mu}$, which is fixed to grain body, moves together with the grain and thus radiates electric dipole emission.

4.2. Power Spectrum of a Freely Spinning Grain. Consider a grain with a dipole moment $\boldsymbol{\mu}$ fixed in the grain body rotating with an angular momentum J. If the grain only spins around its symmetry axis, then the rotating dipole moment emits radiation at a unique frequency $v$ equal to the rotational frequency, that is, $\nu=\omega / 2 \pi$ (see DL98). The power spectrum for this case is simply a delta function $\delta(\nu-\omega / 2 \pi)$ with a unique frequency mode.

For an irregular grain of triaxial ellipsoid shape, the grain rotational dynamics is more complicated. In general, one can also obtain analytical expressions for power spectrum, but it is rather tedious. To find the power spectrum of a freely rotating irregular grain, Hoang et al. $[13,15]$ have employed a more simple brute force approach based on the Fourier transform approach. First, they represent the dipole moment $\boldsymbol{\mu}$ in an inertial coordinate system, and then they compute its second derivative. We obtain

$$
\ddot{\mu}=\sum_{i=1}^{3} \mu_{i} \ddot{\mathbf{a}}_{i}
$$

where $\mu_{i}$ are components of $\boldsymbol{\mu}$ along principal axes $\mathbf{a}_{i}, \ddot{\mathbf{a}}_{i}$ are second derivatives of $\mathbf{a}_{i}$ with respect to time, and $i=1,2$, and 3.

The instantaneous emission power by the rotating dipole moment is equal to

$$
P_{\mathrm{ed}}(J, q, t)=\frac{2}{3 c^{3}} \ddot{\mu}^{2} .
$$






(a)



(b)

FIgURE 2: Normalized power spectrum of a torque-free rotating irregular grain with $I_{1}: I_{2}: I_{3}=1: 0.6: 0.5$ for the different values of $q=1.05,1.60$ (i.e., $\left.q<q_{\mathrm{sp}} \equiv I_{1} / I_{2}\right)$ and $q=1.81>q_{\mathrm{sp}}$. The components of $\left|\mathrm{FT}\left(\ddot{\mu}_{x}\right)\right|^{2} / \max \left(\left|\mathrm{FT}\left(\ddot{\mu}_{x}\right)\right|^{2}\right)\left(\operatorname{or}\left|\mathrm{FT}\left(\ddot{\mu}_{y}\right)\right|^{2} / \max \left(\left|\mathrm{FT}\left(\ddot{\mu}_{x}\right)\right|^{2}\right)\right.$ are indicated by circles, while the components of $\left|\mathrm{FT}\left(\ddot{\mu}_{z}\right)\right|^{2} / \max \left(\left|\mathrm{FT}\left(\ddot{\mu}_{x}\right)\right|^{2}\right)$ are indicated by triangles. Orders of in-plane modes $m$ and out-of-plane modes $n$ are indicated, and case $1\left(\mu_{1}=\mu / \sqrt{3}\right)$ of $\boldsymbol{\mu}$ orientation is assumed. The figure is reproduced from Hoang et al. [15].

The power spectrum is then obtained from the Fourier transform (FT) for the components of $\ddot{\mu}$. For example, the amplitude of $\ddot{\mu}_{x}$ at the frequency $v_{k}$ is defined as

$$
\ddot{\mu}_{x, k}=\int_{-\infty}^{+\infty} \ddot{\mu}_{x}(t) \exp \left(-i 2 \pi v_{k} t\right) d t
$$

where $k$ denotes the frequency mode. The emission power at the positive frequency $v_{k}$ is given by

$$
P_{\mathrm{ed}, k}(J, q)=\frac{4}{3 c^{3}}\left(\ddot{\mu}_{x, k}^{2}+\ddot{\mu}_{z, k}^{2}+\ddot{\mu}_{z, k}^{2}\right),
$$

where the factor 2 arises from the positive/negative frequency symmetry of the Fourier spectrum. To reduce the spectral leakage in the FT, we convolve the time-dependent function $\ddot{\mu}$ with the Blackman-Harris window function (see Harris, 1978). The power spectrum then needs to be corrected for the power loss due to the window function.

The total emission power from all frequency modes for a given $J$ and $q$ then becomes

$$
P_{\mathrm{ed}}(J, q)=\sum_{k} P_{\mathrm{ed}, k}(J, q) \equiv \frac{1}{T} \int_{0}^{T} d t\left(\frac{2}{3 c^{3}} \ddot{\mu}^{2}\right),
$$

where $T$ is the integration time. (this is the result of Parseval's theorem).

Figure 2 presents normalized power spectra (squared amplitude of Fourier transforms), $\left|\mathrm{FT}\left(\mu_{x, y}\right)\right|^{2 /}$ $\max \left(\left|\mathrm{FT}\left(\mu_{x}\right)\right|^{2}\right)$ and $\left|\mathrm{FT}\left(\mu_{z}\right)\right|^{2} / \max \left(\left|\mathrm{FT}\left(\mu_{x}\right)\right|^{2}\right)$, for the components $\ddot{\mu}_{x}$ (or $\ddot{\mu}_{y}$ ) and $\ddot{\mu}_{z}$ for a freely rotating irregular grain having the ratio of moments of inertia $I_{1}: I_{2}: I_{3}=1: 0.6: 0.5$ and for various $q$. Circles and triangles indicated with $m$ and $n$ denote peaks of the power spectrum for oscillating components of $\ddot{\mu}_{x}$ (or $\ddot{\mu}_{y}$ ) and $\ddot{\mu}_{z}$, respectively. The horizontal axis is the angular frequency of emission modes normalized over the frequency of emission when the grain spins around its shortest axis.
Multiple frequency modes are observed in the power spectra of the irregular grain, but in Figure 2, we show only the modes with power no less than $10^{-3}$ the maximum value. One can see that in the case with large $q=1.6$, the modes with $\omega /\left(J / I_{1}\right)>1$ have increasing power, while the modes with $\omega /\left(J / I_{1}\right)<1$ have decreasing power. It indicates that if grain rotational energy is increased so that the grain spends a significant fraction of time rotating with large $q$, then the grain should radiate larger rotational emission.

Although one should not expect the analytical expression of power spectrum for the triaxial grain, the frequency modes can be approximately found. Indeed, for $q<I_{1} / I_{2}$, we found that power spectra for $\ddot{\mu}_{x}$ (or $\ddot{\mu}_{y}$ ) have angular frequency modes

$$
\omega_{m} \approx\langle\dot{\phi}\rangle+m\langle|\dot{\psi}|\rangle
$$

where the bracket denotes the averaging value over time, and $m=0, \pm 1, \pm 2 \ldots$ denote the order of the mode. The frequency modes for $\ddot{\mu}_{z}$ are given by

$$
\omega_{n}=n\langle|\dot{\psi}|\rangle,
$$

where $n$ is integer and $n \geq 1$.

In the following, the emission modes induced by the oscillation of $\mu_{x}$ or $\mu_{y}$, which lie in the $\hat{\mathbf{x}} \hat{\mathbf{y}}$ plane, perpendicular to $\mathbf{J}$, are called in-plane modes, and those induced by the oscillation of $\mu_{z}$ in the direction perpendicular to the $\widehat{\mathbf{x}} \hat{\mathbf{y}}$ plane, are called out-of-plane modes. The order of mode is denoted by $m$ and $n$, respectively. Figure 2 also shows that the emission power for out-of-plane modes $\omega_{n}$ is negligible compared to the power emitted by in-plane modes $\omega_{m}$.

Emission power spectra are numerically calculated to find $\omega_{k}$ and $P_{\mathrm{ed}, k}$, as functions of $J$ and $q$, for the various ratio of moments of inertia $I_{1}: I_{2}: I_{3}$. The obtained data will be used later to compute spinning dust emissivity. 


\section{Grain Angular Momentum Distribution: Langevin Equation}

5.1. Langevin Equation. To find the exact distribution function for grain angular momentum J, Hoang et al. [13] and Hoang et al. [15] proposed a numerical approach based on the Langevin equation. Basically, they numerically solved the Langevin equation describing the evolution of three components of $\mathbf{J}$ in an inertial coordinate system. They read

$$
d J_{i}=A_{i} d t+\sqrt{B_{i i}} d q_{i}, \quad \text { for } i=x, y, z,
$$

where $d q_{i}$ are random Gaussian variables with $\left\langle d q_{i}^{2}\right\rangle=d t$, and $A_{i}=\left\langle\Delta J_{i} / \Delta t\right\rangle$ and $B_{i i}=\left\langle\left(\Delta J_{i}\right)^{2} / \Delta t\right\rangle$ are damping and diffusion coefficients defined in the inertial coordinate system. Detailed expressions of these coefficients can be found in Hoang et al. [13] and Hoang et al. [15].

For an irregular grain, and to simplify calculations, we adopt the $A_{i}$ and $B_{i i}$ for a disk-like grain obtained in HDL10. Following DL98b and HDL10, the disk-like grain has the radius $R$ and thickness $L=3.35 \AA$, and the ratio of moments of inertia is along and perpendicular to the grain symmetry axis $h=I_{\|} / I_{\perp}$. Thereby, the effect of nonaxisymmetry on $A_{i}$ and $B_{i i}$ is ignored, and we only examine the effect of grain wobbling resulting from the grain triaxiality.

In dimensionless units, $\mathbf{J}^{\prime} \equiv \mathbf{J} / I_{\|} \omega_{T, \|}$ with $\omega_{T, \|} \equiv$ $\left(2 k_{B} T_{\text {gas }} / I_{\|}\right)^{1 / 2}$ being the thermal angular velocity of the grain along the grain symmetry axis and $t^{\prime} \equiv t / \tau_{H, \|} ;(14)$ becomes

$$
d J_{i}^{\prime}=A_{i}^{\prime} d t^{\prime}+\sqrt{B_{i i}^{\prime}} d q_{i}^{\prime},
$$

where $\left\langle d q_{i}^{2}\right\rangle=d t^{\prime}$,

$$
\begin{gathered}
A_{i}^{\prime}=-\frac{J_{i}^{\prime}}{\tau_{\mathrm{gas}, \mathrm{eff}}^{\prime}}-\frac{2}{3} \frac{J_{i}^{\prime 3}}{\tau_{\mathrm{ed}, \mathrm{eff}}^{\prime}}, \\
B_{i i}^{\prime}=\frac{B_{i i}}{2 I_{\|} k_{\mathrm{B}} T_{\mathrm{gas}}} \tau_{H, \|},
\end{gathered}
$$

where

$$
\begin{gathered}
\tau_{\text {gas,eff }}^{\prime}=\frac{\tau_{\text {gas,eff }}}{\tau_{H, \|}}=\frac{F_{\text {tot }, \|}^{-1}}{\cos ^{2} \theta+\gamma_{H} \sin ^{2} \theta}, \\
\gamma_{H}=\frac{F_{\text {tot, } \perp} \tau_{H, \|}}{F_{\text {tot }, \|} \tau_{H, \perp}}, \quad \tau_{\text {ed,eff }}^{\prime}=\frac{\tau_{\text {ed,eff }}}{\tau_{H, \|}},
\end{gathered}
$$

where $\tau_{H, \|}$ and $\tau_{H, \perp}$ are rotational damping times due to gas of purely hydrogen atom for rotation along parallel and perpendicular direction to the grain symmetry axis $\mathbf{a}_{1}, \tau_{\text {ed,eff }}$ is the effective damping time due to electric dipole emission (see HDL10, HLD11), $\theta$ is the angle between $\mathbf{a}_{1}$ and $\mathbf{J}$, and $F_{\text {tot, } \|}$ and $F_{\text {tot, } \perp}$ are total damping coefficients parallel and perpendicular to $\mathbf{a}_{1}$ (see HDL10). In the case of fast internal relaxation, the diffusion coefficients $A$ and $B$ are averaged over the distribution function $f_{\mathrm{VRE}}$.

The Langevin equation (15) is solved using the numerical integration with a constant timestep. At each timestep, the angular momentum $J_{i}$ obtained from LEs is recorded and later used to find the distribution function $f_{J}$ with normalization $\int_{0}^{\infty} f_{J} d J=1$.
5.2. Advantages of the Langevin Equation Approach. There are two apparent advantages of the LE approach. First, it allows us to treat the spinning dust emission from grains with an arbitrary grain vibrational temperature. Second, the impulsive excitation by single-ion collisions, which can deposit an amount of angular momentum greater than the grain angular momentum prior the collision, is easily included in (14) (see [15]). Next, we briefly discuss the effect of impulsive excitations arising from single-ion collisions.

DL98b showed that for grains smaller than $7 \AA$, the angular impulse due to an individual ion-grain collision may be comparable to the grain angular momentum prior the collision. Thus, infrequent hits of ions can result in the transient rotational excitation for very small grains.

Let $\tau_{i \text { coll }}^{-1}$ be the mean rate of ion collisions with the grain given by

$$
\begin{aligned}
\tau_{\text {icoll }}^{-1}= & f\left(Z_{g}=0\right) n_{i} \pi a^{2}\left(\frac{8 k_{B} T_{\text {gas }}}{m_{i} \pi}\right)^{1 / 2}\left[1+\frac{\sqrt{\pi}}{2} \Phi\right] \\
& +\sum_{Z_{g} \neq 0} f\left(Z_{g}\right) n_{1} \pi a^{2}\left(\frac{8 k_{B} T_{\text {gas }}}{m_{i} \pi}\right)^{1 / 2} g\left(\frac{Z_{g} Z_{i} e^{2}}{a k_{B} T_{\text {gas }}}\right),
\end{aligned}
$$

where $\Phi=\left(2 Z_{i}^{2} e^{2} / a k_{B} T_{\text {gas }}\right)^{1 / 2}, g(x)=1-x$ for $x<0$ and $g(x)=e^{-x}$ for $x>0$, and $f\left(Z_{g}\right)$ is the grain charge distribution function. The probability of the next collision occurring in $[t, t+d t]$ is

$$
d P=\tau_{\text {icoll }}^{-1} \exp \left(\frac{-t}{\tau_{\text {icoll }}}\right) d t
$$

The rms angular momentum per ion collision $\left\langle\delta J^{2}\right\rangle$ is inferred by dividing the total rms angular momentum by the collision rate, and its final formula is given in Hoang et al. [13].

Provided that the random moment of a single-ion collision is obtained from (19), the angular momentum that the grain acquires through each single-ion collision can easily be incorporated into the Langevin equation (14). Hoang et al. [13] found that the impulsive excitations of ions extend the distribution of grain angular momentum to the region of high angular momentum (see next section for its effect on spinning dust emission).

\section{Spinning Dust Emissivity}

6.1. Spinning Grain of Triaxial Ellipsoid Shape. An irregular grain rotating with a given angular momentum $J$ radiates at frequency modes $\omega_{k} \equiv \omega_{m}$ with $m=0, \pm 1, \pm 2 \ldots$ and $\omega_{k} \equiv$ $\omega_{n}$ with $n=1,2,3 \ldots$ (see (12) and (13)). For simplicity, let $\omega_{m_{i}}$ denote the former and $\omega_{n_{i}}$ denote the latter where $i$ indicates the value for $m$ and $n$. These frequency modes depend on the parameter $q(s)$, which is determined by the internal thermal fluctuations within the grain.

To find the spinning dust emissivity by a grain at an observational frequency $v$, first we need to know how much emission that is contributed by each mode $\omega_{k}$. 
Consider an irregular grain rotating with the angular momentum $J$; the probability of finding the emission at the angular frequency $\omega$ depends on the probability of finding the value $\omega$ such that

$$
p d f(\omega \mid J) d \omega=f_{\mathrm{VRE}}(s, J) d s=A \exp \left(-\frac{q(s) J^{2}}{2 I_{1} k_{B} T_{\mathrm{vib}}}\right) d s,
$$

where we assumed the VRE regime with $f_{\mathrm{VRE}}$ given by (6).

For the mode $\omega \equiv \omega_{k}(s)$, from (20) we can derive

$$
p d f_{k}(\omega \mid J)=\left(\frac{\partial \omega_{k}}{\partial s}\right)^{-1} f_{\mathrm{VRE}}(s, J)
$$

The emissivity from the mode $k$ is calculated as

$$
\begin{aligned}
j_{\nu, k}^{a}= & \frac{1}{4 \pi} \int_{J_{l}}^{J_{u}} P_{\mathrm{ed}, k}\left(J, q_{\leq}\right) f_{J}(J) p d f_{k}(\omega \mid J) 2 \pi d J \\
& +\frac{1}{4 \pi} \int_{J_{l}}^{J_{u}} P_{\mathrm{ed}, k}\left(J, q_{>}\right) f_{J}(J) p d f_{k}(\omega \mid J) 2 \pi d J,
\end{aligned}
$$

where $q_{\leq}$and $q>$ denote $q \leq q_{\mathrm{sp}}$ and $q>q_{\mathrm{sp}}$, respectively, $J_{l}$ and $J_{u}$ are lower and upper limits for $J$ corresponding to a given angular frequency $\omega_{k}(J, q)=\omega$, and $2 \pi$ appears due to the change of variable from $v$ to $\omega$.

Emissivity by a grain of size $a$ at the observation frequency $v$ arising from all emission modes is then

$$
j_{v}^{a} \equiv \sum_{k} j_{\nu, k}^{a}
$$

Consider, for example, the emission mode $k \equiv m_{0}$. For the case $I_{2}$ which is slightly larger than $I_{3}$, this mode has the angular frequency $\omega_{m_{0}}=\langle\dot{\phi}\rangle=\left(J / I_{1}\right) q_{0}$ with $q_{0}$ obtained from calculation of $\omega_{m_{0}}$, which is independent of $q$ for $q<q_{\mathrm{sp}}$ $\left(q_{0}\right.$ approaches $I_{1} / I_{2}$ as $I_{3} \rightarrow I_{2}$, i.e., when irregular shape becomes spheroid). As a result,

$$
p d f_{m_{0}}(\omega \mid J)=\delta\left(\omega-\left(\frac{J}{I_{1}}\right) q_{0}\right) .
$$

Thus, the first term of (22), denoted by $j_{v, \mathrm{~m}_{0}, \leq}^{a}$, is rewritten as

$$
\begin{aligned}
j_{\gamma, m_{0}, \leq}^{a} & =\frac{1}{2} \int_{J_{l}}^{J_{u}} P_{\mathrm{ed}, m_{0}}\left(J, q_{\leq}\right) f_{J}(J) \delta\left(\omega-\left(\frac{J}{I_{1}}\right) q_{0}\right) d J \\
& =\frac{1}{2} \frac{I_{1} f_{J}\left(J_{0}\right)}{q_{0}} P_{\mathrm{ed}, m_{0}}\left(J_{0}, q(s)\right),
\end{aligned}
$$

where $J_{0}=I_{1} \omega / q_{0}$, and the value of $q(s)$ remains to be determined.

For $q>q_{\mathrm{sp}},\langle\dot{\phi}\rangle$ is a function of $q$. Hence, the emissivity (22) for the mode $k \equiv m_{0}$ becomes

$$
\begin{aligned}
j_{\nu, m_{0}}^{a}= & \frac{1}{2} \frac{I_{1} f_{J}\left(J_{0}\right)}{q_{0}} \int_{0}^{s_{s p}} d s P_{\mathrm{ed}, m_{0}}\left(J_{0}, q(s)\right) f_{\mathrm{VRE}}\left(J_{0}, s\right) \\
& +\frac{1}{2} \int_{J_{l}}^{J_{u}} P_{\mathrm{ed}, m_{0}}\left(J, q_{>}\right) f_{J}(J) p d f_{m_{0}}(\omega \mid J) d J,
\end{aligned}
$$

where $s_{\mathrm{sp}}$ is the value of $s$ corresponding to $q=q_{\mathrm{sp}}$, and the term $P_{\text {ed, } m_{0}}\left(J_{0}, q(s)\right)$ in (25) has been replaced by its average value over the internal thermal distribution $f_{\text {VRE }}$.

The emissivity per $H$ is obtained by integrating $j_{v}^{a}$ over the grain size distribution

$$
\frac{j_{v}}{n_{H}}=\frac{1}{n_{H}} \int_{a_{\min }}^{a_{\max }} d a \frac{d n}{d a} j_{\nu}^{a},
$$

where $j_{v}^{a}$ is given by (23).

6.2. A Degenerate Case: Grains of Disk-Like Shape. The spinning dust emissivity from disk-like grains (e.g., $I_{2}=I_{3}$ ) is a degenerate case of triaxial grains. Basically, a disk-like grain with an angular momentum $\mathbf{J}$ radiates at four frequency modes as follows:

$$
\begin{aligned}
& \omega_{m_{i}} \equiv \dot{\phi}+i \dot{\psi}=\frac{J}{I_{\|}}[h+i(1-h) \cos \theta], \\
& \omega_{n_{1}} \equiv \dot{\psi}=\frac{J}{I_{\|}}(1-h) \cos \theta,
\end{aligned}
$$

where $i=0$ and \pm 1 (see HDL10 and [12]).

The emission power of these modes are given by the following analytical forms (HDL10 and [16]):

$$
\begin{aligned}
P_{\omega_{m_{0}}} & =\frac{2 \mu_{\|}^{2}}{3 c^{3}} \omega_{m_{0}}^{4} \sin ^{2} \theta, \\
P_{\omega_{m_{ \pm 1}}} & =\frac{\mu_{\perp}^{2}}{6 c^{3}} \omega_{m_{ \pm 1}}^{4}(1 \pm \cos \theta)^{2}, \\
P_{\omega_{n_{1}}} & =\frac{2 \mu_{\perp}^{2}}{3 c^{3}} \omega_{n_{1}}^{4} \sin ^{2} \theta .
\end{aligned}
$$

For the disk-like grain, from (3), the number of states in phase space $s$ for $q$ spanning from $1-q$ becomes

$$
s=1-\left(\frac{h-q}{h-1}\right)^{1 / 2}=1-\cos \theta,
$$

where $q=1+(h-1) \sin ^{2} \theta$ has been used. Thus, for an arbitrary mode with frequency $\omega_{k}$, we obtain

$$
p d f_{k}(\omega \mid J) d \omega=f_{\mathrm{VRE}}(s, J) d s=f_{\mathrm{VRE}}(\theta, J) \sin \theta d \theta .
$$

Taking use of $\omega=\omega_{k}(J, \theta)$, we derive

$$
p d f_{k}(\omega \mid J)=f_{\mathrm{VRE}}(\theta, J)\left(\frac{\partial \omega_{k}}{\partial \theta}\right)^{-1} \sin \theta .
$$

Therefore, by substituting (29) in (22), the emissivity at the observation frequency $v=\omega /(2 \pi)$ from a disk-like grain of size $a$ is now given by

$$
\begin{aligned}
j_{v}^{a} \equiv & \frac{1}{2} \frac{f_{J}\left(I_{\|} \omega / h\right)}{h} \frac{2 \mu_{\|}^{2}}{3 c^{3}} \omega^{4}\left\langle\sin ^{2} \theta\right\rangle \\
& +\frac{1}{2} \frac{\mu_{\perp}^{2}}{6 c^{3}} \omega^{4} \int_{J_{l}}^{J_{u}} p d f_{m_{1}}(\omega \mid J) f_{J}(J) d J \\
& +\frac{1}{2} \frac{\mu_{\perp}^{2}}{6 c^{3}} \omega^{4} \int_{J_{l}}^{J_{u}} p d f_{m_{-1}}(\omega \mid J) f_{J}(J) d J \\
& +\frac{1}{2} \frac{\mu_{\perp}^{2}}{3 c^{3}} \omega^{4} \int_{J_{l}}^{I_{u}} p d f_{n_{1}}(\omega \mid J) f_{J}(J) d J,
\end{aligned}
$$




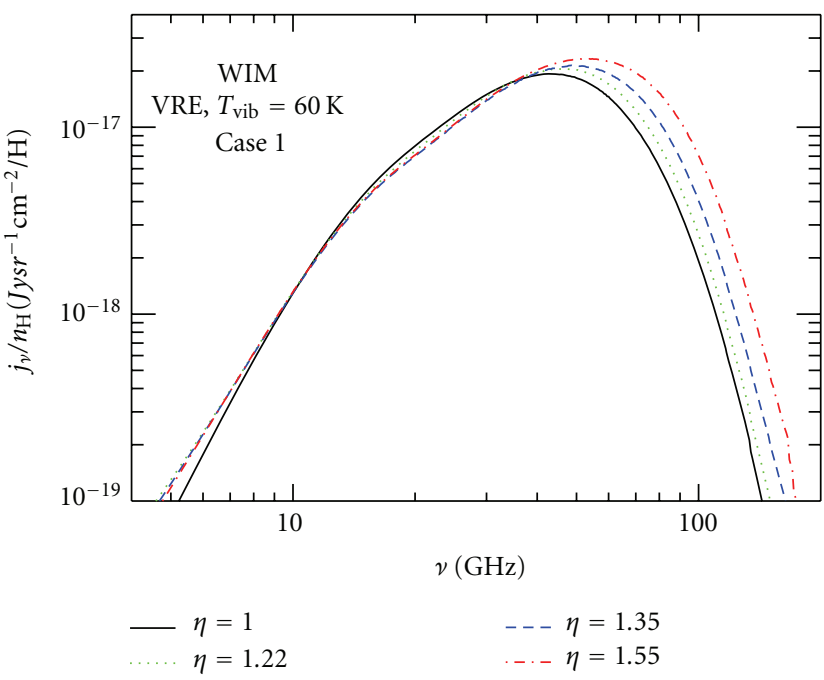

(a)

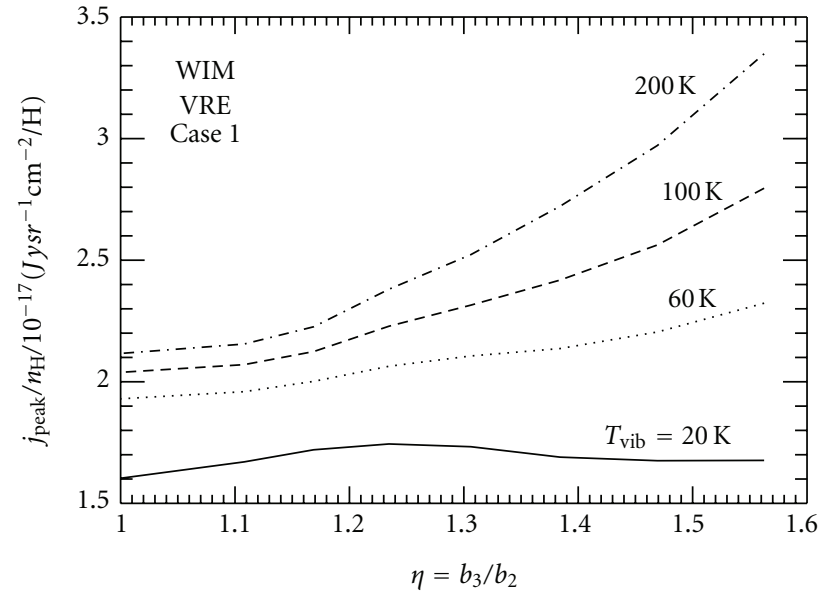

(b)

FIgURE 3: Emissivity per $H$ from irregular grains of different degrees of irregularity $\eta=b_{3} / b_{2}$ with $T_{\text {vib }}=60$ and $200 \mathrm{~K}$ in the WIM for the case in which the electric dipole moment is isotropically oriented in the grain body (i.e., case 1 with $\mu_{1}=\mu / \sqrt{3}$ ). The emission spectrum shifts to higher frequency as $\eta$ decreases (i.e., grain becomes more irregular). Here, the grain mass is held fixed as $\eta$ changes. The figure is reproduced from Hoang et al. [15].

where $p d f_{m_{ \pm 1}}$ and $p d f_{n_{1}}$ are easily derived by using (32) for $\omega_{m_{ \pm 1}}$ and $\omega_{n_{1}}$, and $J_{l}=I_{\|} \omega /(2 h-1)$ and $J_{u}=I_{\|} \omega$ for $m_{ \pm 1}$ mode, $J_{l}=I_{\|} \omega /(h-1)$ and $J_{u}=\infty$ for $n_{1}$ mode.

6.3. Emissivity. Hoang et al. [15] assumed that the smallest grains of size $a \leq a_{2}=6 \AA$ have irregular shape and larger grains are spherical. To compare the emissivity from an irregular grain with that from a disk-like grain, they considered the simplest case of the irregular shape in which the circular cross section of the disk-like grain is adjusted to the elliptical cross section. The emission by two grains of different shapes with the same mass $M$ and thickness $L$ is under interest; therefore, the semiaxes of the elliptical disk are constrained by the grain mass

$$
M=\pi R^{2} L=\pi b_{2} b_{3} L
$$

where $R=\left(4 a^{3} / 3 L\right)^{1 / 2}$ is the radius of the disk-like grain, $b_{2}$ and $b_{3}$ are the length of semiaxes $\mathbf{a}_{2}$ and $\mathbf{a}_{3}$, and $b_{1}=L$ is kept constant. Assuming that the circular disk is compressed by a factor $\alpha \leq 1$ along $\mathbf{a}_{2}$, then (34) yields

$$
b_{2}=\alpha R, \quad b_{3}=\alpha^{-1} R
$$

Denote the parameter by $\eta \equiv b_{3} / b_{2}=\alpha^{-2}$, then the degree of grain shape irregularity is completely characterized by $\eta$.

For each grain size $a$, the parameter $\eta$ is increased from $\eta=1$ to $\eta=\eta_{\max }$. However, $\eta_{\max }$ is constrained by the fact that the shortest axis $\mathbf{a}_{2}$ should not be shorter than the grain thickness $L$. The value $\eta_{\max } \sim 3 / 2$ is conservatively chosen.

Although the irregular grain can radiate at a large number of frequency modes, only the modes with the order $|m| \leq 2$ are important. The higher-order modes contribute less than $\sim 0.5 \%$ to the total emission, and thus they are neglected. Hoang et al. [15] assumed that grains smaller than $a_{2}$ have a fixed vibrational temperature $T_{\text {vib }}$ (see Hoang et al. [15] for the detailed treatment of $T_{\text {vib }}$ distribution), and that for the instantaneous value of $J$, the rotational energy has a probability distribution $f_{\mathrm{VRE}}$ (i.e., VRE regime, see (6)).

The grain size distribution $d n / d a$ from Draine and $\mathrm{Li}$ [42] is adopted with the total to selective extinction $R_{V}=3.1$ and the total carbon abundance per hydrogen nucleus $b_{C}=$ $5.5 \times 10^{-5}$ in carbonaceous grains with $a_{\min }=3.55 \AA$ and $a_{\text {max }}=100 \AA$.

The spinning dust emissivity is calculated for a so-called model A (similar to DL98b; HDL10), in which 25\% of grains have the electric dipole moment parameter $\beta=2 \beta_{0}, 50 \%$ have $\beta=\beta_{0}$, and $25 \%$ have $\beta=0.5 \beta_{0}$ with $\beta_{0}=0.4 \mathrm{D}$. In the rest of the paper, the notation model $\mathrm{A}$ is omitted, unless stated otherwise.

The left panel in Figure 3 shows the spinning dust emissivity for different degrees of irregularity $\eta$ and with a dust temperature $T_{\text {vib }}=60 \mathrm{~K}$ in the WIM. The emission spectrum for a given $T_{\text {vib }}$ shifts to higher frequency as $\eta$ decreases (i.e., the degree of grain irregularity increases), but their spectral profiles remain similar. The right panel shows the increase of peak emissivity $J_{\text {peak }}$ with increasing $\eta$.

One particular feature in Figure 3(b) is that for axisymmetric grains $(\eta=1)$, the emissivity increases by a factor of 1.3 with $T_{\text {vib }}$ increasing from 20 to $200 \mathrm{~K}$. However, for the irregular grain with high triaxiality $\eta=1.5$, the emissivity increases by a factor of 2 . The peak frequency is increased by a factor of 1.4.

This feature is easy to understand because the irregular grain radiate at more frequency modes than the axisymmetric grain. As a result, for the grain temperature to increase to 


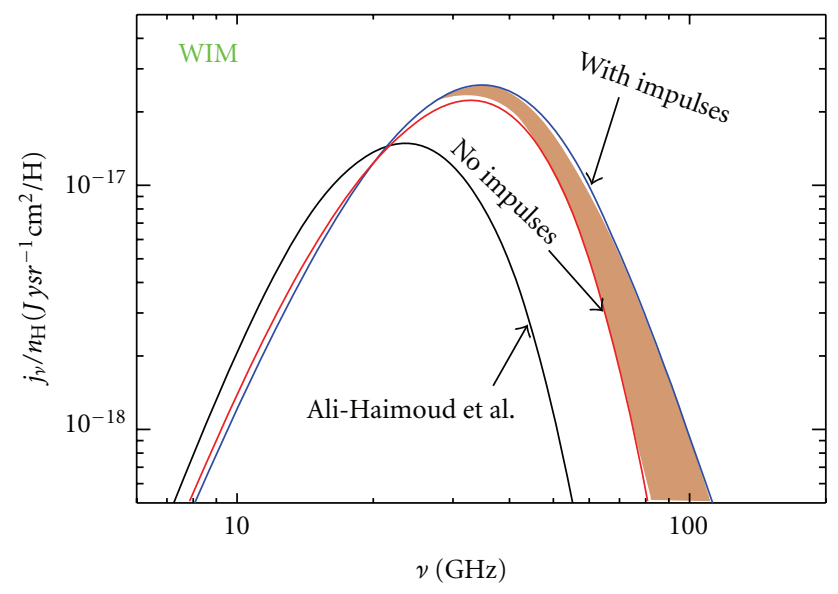

FIgURE 4: Emissivity per $H$ obtained for WIM without ionic impulses using the Fokker-Planck equation from Ali-Haïmoud et al. [12] and with impulses using our LE simulations for grain wobbling. The spectra are efficiently broadened as a result of impulses (see blue line). The figure is reproduced from Hoang et al. [13].

a sufficiently high value, it results in the uniform distribution of the angle between grain symmetry axis and angular momentum, so that the spinning dust emissivity becomes saturated. On the other hand, for the triaxial grain, as $T_{\text {vib }}$ increases, it allows the grain to rotate about its axis of minimum inertia (smallest moment of inertia). As a result, the grain radiates at frequency modes with higher frequency and power.

In the case of efficient IVRET, vibrational energy is converted to rotational emission, which results in the increase of both emissivity and peak frequency. As shown, the energy transfer is more efficient for the more irregular grain. The reason for this is that the more irregular grain allows the grain to spend a larger fraction of time rotating along the axis of minor inertia.

The effect of impulsive excitations by single-ion collisions is shown in Figure 4. One can see that the impulses from ions can increase the emissivity by $\sim 23 \%$ and slightly increase the peak frequency (see Figure 4). The tail of high frequency part is obviously extended due to the contribution from ionic impulses with large angular momentum.

\section{Constraining Spinning Dust Parameters and Implications}

Spinning dust emission involves a number of parameters, including grain physical parameters and environmental parameters. Among them, the grain dipole moment and gas density are two most important parameters, but they can be constrained using theoretical modeling combined with observation data (see, e.g., Dobler et al. [43] and Hoang et al. [15]). In the following, we discuss a number of parameters, which are shown to be important but more difficult to constrain through observation.

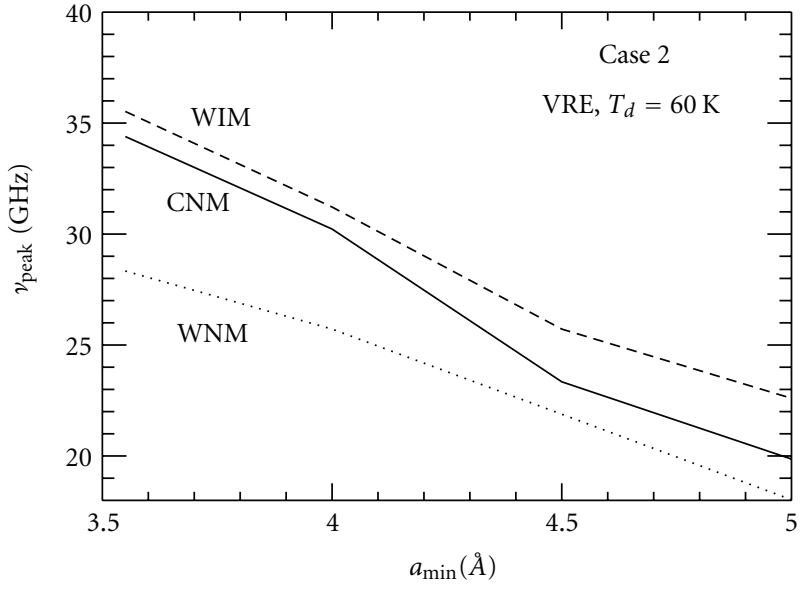

FIGURE 5: Decrease of the peak frequency $\nu_{\text {peak }}$ of spinning dust spectrum with the lower cutoff of grain size distribution $a_{\min }$ for various environmental conditions. The figure is reproduced from Hoang et al. [15].

7.1. Lower Cutoff of Grain Size Distribution $a_{\min }$. The spinning dust emission spectrum is sensitive to the population of tiny dust grains, and its peak frequency is mostly determined by the smallest PAHs. Let $a_{\min }$ be the size of the smallest PAHs. When $a_{\min }$ is increased, the peak frequency $v_{\text {peak }}$ decreases accordingly.

Figure 5 shows the variation of $v_{\text {peak }}$ as a function of $a_{\min }$ for various environments for the case in which the grain dipole moment lies in the grain plane (Case 2) with $\mu_{1}=0$ and with the VRE regime $\left(T_{d}=60 \mathrm{~K}\right)$. As expected, $v_{\text {peak }}$ decreases generically with $a_{\min }$ increasing. Thus, in addition to grain dipole moment, the lower cutoff of grain size also plays an important role.

7.2. Constraining the Shape of Very Small Grains. Very small grains and PAHs are expected to be nonspherical. However, constraining grain triaxiality using spinning dust appears rather challenging. In the simplest case where the grain shape can be approximated as a triaxial ellipsoid, the possibility is still low because there are many parameters involved in the spinning dust.

7.3. Can Compressible Turbulence Be Observed through Spinning Dust Emission? The discussion of interstellar conditions adopted in DL98 and other works on spinning dust was limited by idealized interstellar phases. It is now recognized that turbulence plays an important role in shaping the interstellar medium.

For spinning dust, the turbulence can increase the emissivity due to its nonlinear dependence on material density. Indeed, in a medium with density fluctuations, the effective emissivity is

$$
\left\langle j_{v}\right\rangle=\int_{0}^{1} f(x) j_{v}(x\langle\rho\rangle) d x,
$$

where $f(x) d x$ is the fraction of the mass with $\rho /\langle\rho\rangle \in(x, x+$ $d x)$. We use compression distributions $f(x)$ obtained from 


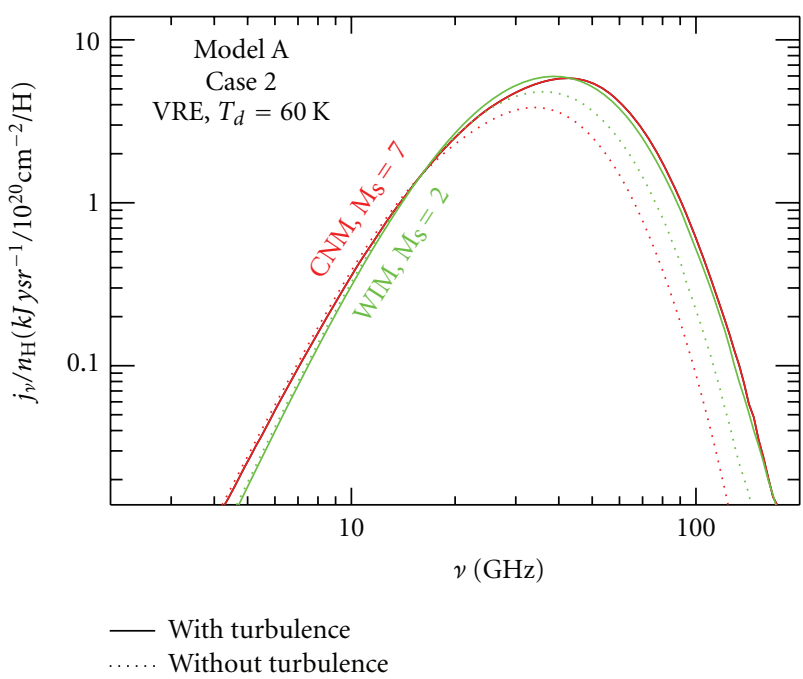

Figure 6: Spinning dust emissivity per $H$ in the presence of compressible turbulence with sonic Mach number $M_{s}=2$ and 7, compared to that from uniform medium with $n_{H}=\bar{n}_{H}$ for the CNM (red) and WIM (green). The peak emissivity is increased, and the spectrum is shifted to higher frequency due to compressible turbulence. Case $2\left(\mu_{1}=0\right)$ of $\boldsymbol{\mu}$ orientation is considered. Figure reproduced from Hoang et al. [15].

MHD simulations for $M_{s}=2$ and 7 to evaluate $\left\langle j_{v}\right\rangle$ for the WIM and CNM, respectively.

We assume the case $2\left(\mu_{1}=0\right)$ of $\boldsymbol{\mu}$ orientation. The resulting effective emissivity is compared with the emissivity from the uniform medium in Figure 6. It can be seen that the turbulent compression increases the emissivity and shifts the peak to higher $v_{\text {peak }}$. The increase of emissivity is significant for strong turbulent medium.

The distribution of phases, for instance, CNM and WNM of the ISM at high latitudes, can be obtained from absorption lines. Similarly, by studying fluctuations of emission, it is possible to constrain parameters of turbulence. In an idealized case of a single-phase medium with fluctuations of density with a given characteristic size, one can estimate the value of the 3D fluctuation by studying the 2D fluctuations of column density. More sophisticated techniques for obtaining sonic Mach numbers have been developed recently (see Kowal et al. [44], Esquivel and Lazarian [45] and Burkhart et al. [46]). (It may be seen that Alfven Mach numbers have subdominant effect on the distribution of densities (see Kowal et al. [44]). Thus, in our study we did not vary the Alfven Mach number.) In particular, Burkhart et al. [47], using just column density fluctuations of the SMC, obtained a distribution of Mach numbers corresponding to the independent measurements obtained using Doppler shifts and absorption data. With such an input, it is feasible to quantify the effect of turbulence in actual observational studies of spinning dust emission.

7.4. Effect of Dust Acceleration on Spinning Dust Emission. Collisions of ultrasmall grains with ions and neutrals in plasma appear to be a dominant mechanism of rotational excitations for spinning dust emission, particularly, in dark clouds where UV photons are blocked out. Current spinning dust models assume Brownian motion of grains relative to gas, but it is known that grains may move with suprathermal velocities due to acceleration by turbulence (see, e.g., [48], Yan and Lazarian $[49,50]$, and Hoang et al. [51]) and random charge fluctuations (Ivlev et al. [52] and Hoang and Lazarian [53]). The latter mechanism, namely, random charge fluctuations-induced acceleration, is found to be efficient for tiny grains (Hoang and Lazarian [53]).

The resonant acceleration by fast modes of MHD turbulence, which occurs when the grain gyroradius is comparable to the scale of turbulence eddy (i.e., $r_{g} \sim$ $\left.k^{-1}\right)$, is considered a dominant mechanism for large grains $\left(>10^{-5} \mathrm{~cm}\right.$ ), whereas it is negligible for ultrasmall grains because the grain gyroradius falls below the cutoff scale of the turbulence due to viscous damping (see Yan et al. [50] and Hoang et al. [51]).

In highly ionized media (e.g., WIM, HII regions), the resonant acceleration by MHD turbulence may become important for ultrasmall grains because the damping cutoff of MHD turbulence is suppressed due to the decrease of viscous neutral damping. We also note that recent observations by Paladini et al. [54]) revealed that PAHs and ultrasmall grains may be present in HII regions, as shown through their $8 \mu \mathrm{m}$ and $24 \mu \mathrm{m}$ emission features, respectively.

Thus, assuming that grain rotational kinetic energy is equal to its translational energy, the acceleration by these aforementioned processes is expected to increase the spinning dust emission. Further studies should take this issue into account.

\section{Polarization of Spinning Dust Emission and Alignment of Ultrasmall Grains}

8.1. Polarization of Anomalous Microwave Emission. Spinning dust emission is an important foreground component that contaminates with the $\mathrm{CMB}$ radiation in the frequency 10-90 GHz. An understanding of how much is this emission component polarized is becoming a pressing question for future CMB B-mode missions.

Recent observational studies (Dickinson et al. [55], López-Caraballo et al. [56], and Macellari et al. [57]) showed that the average polarization of AME is between 2 and $5 \%$. In the last years, significant progress has been made in understanding spinning dust emission, both in theory and observation, but the principal mechanism of alignment of ultrasmall grains is not well understood.

8.2. Alignment of Ultrasmall Dust Grains. Grain alignment is an exciting problem (see Lazarian [58] for a review). The most promising mechanism for the grain alignment is based on radiative torques. Proposed originally by Dolginov and Mytrophanov [59], it is related to the interaction of unpolarized radiation with irregular grains. The numerical studies in Draine and Weingartner $[60,61]$ showed the efficiency and promise of the radiative torques (which later were termed RATs). The physical picture of the RAT 
alignment and a detailed study of important relevant effects are presented in Lazarian and Hoang $[62,63]$ and Hoang and Lazarian [64-66]. However, the efficiency of RATs plummets as the size of grains gets much smaller than the radiation wavelength. Therefore, this mechanism, which seems to provide a good correspondence with the optical and infrared data (see Lazarian [58] and Whittet et al. [67]), cannot be applicable to ultrasmall spinning dust.

Microwave emission from spinning grains is expected to be polarized if grains are aligned. Alignment of ultrasmall grains (essentially PAHs) is likely to be different from alignment of large (i.e., $a>10^{-6} \mathrm{~cm}$ ) grains as discussed previously. One of the mechanisms that might produce the alignment of the ultrasmall grains is the paramagnetic dissipation mechanism proposed by Davis and Greenstein [68]. The Davis-Greenstein alignment mechanism (Davis and Greenstein [68] and Roberge and Lazarian [69]) is straightforward; for a spinning grain, the component of the interstellar magnetic field perpendicular to the grain angular velocity varies in grain coordinates, resulting in timedependent magnetization, associated energy dissipation, and a torque acting on the grain [68]. As a result, grains tend to rotate with angular momenta parallel to the interstellar magnetic field.

Lazarian and Draine [70] (henceforth LD00) found that the traditional picture of paramagnetic relaxation is incomplete, since it disregards the so-called "Barnett magnetization" (Landau and Lifshitz [71]). The Barnett effect, the inverse of the Einstein-de Haas effect, consists of the spontaneous magnetization of a paramagnetic body rotating in field-free space. This effect can be understood in terms of the lattice-sharing part of its angular momentum with the spin system. Therefore, the implicit assumption in Davis and Greenstein [68] that the magnetization within a rotating grain in a static magnetic field is equivalent to the magnetization within a stationary grain in a rotating magnetic field is clearly not exact.

LD00 accounted for the "Barnett magnetization" and termed the effect of enhanced paramagnetic relaxation arising from grain magnetization "resonance paramagnetic relaxation." It is clear from Figure 7 that resonance paramagnetic relaxation persists at the frequencies when the DavisGreenstein relaxation vanishes. However, the polarization is marginal for $v>35 \mathrm{GHz}$ anyhow. The discontinuity at $\sim 20 \mathrm{GHz}$ is due to the assumption that smaller grains are planar and larger grains are spherical. The microwave emission will be polarized in the plane perpendicular to the magnetic field because the angular momentum is partially aligned with the magnetic field.

\subsection{Constraining the Alignment of Ultrasmall Grains}

8.3.1. Can We Constrain the Alignment of Ultrasmall Grains through Polarization of Midinfrared (2-12 $\mu \mathrm{m})$ Emission Features? The answer to this question is "probably not." Indeed, as discussed earlier, midinfrared emission from ultrasmall grains takes place as they absorb UV photons. These photons raise grain vibrational temperature, randomizing grain axes

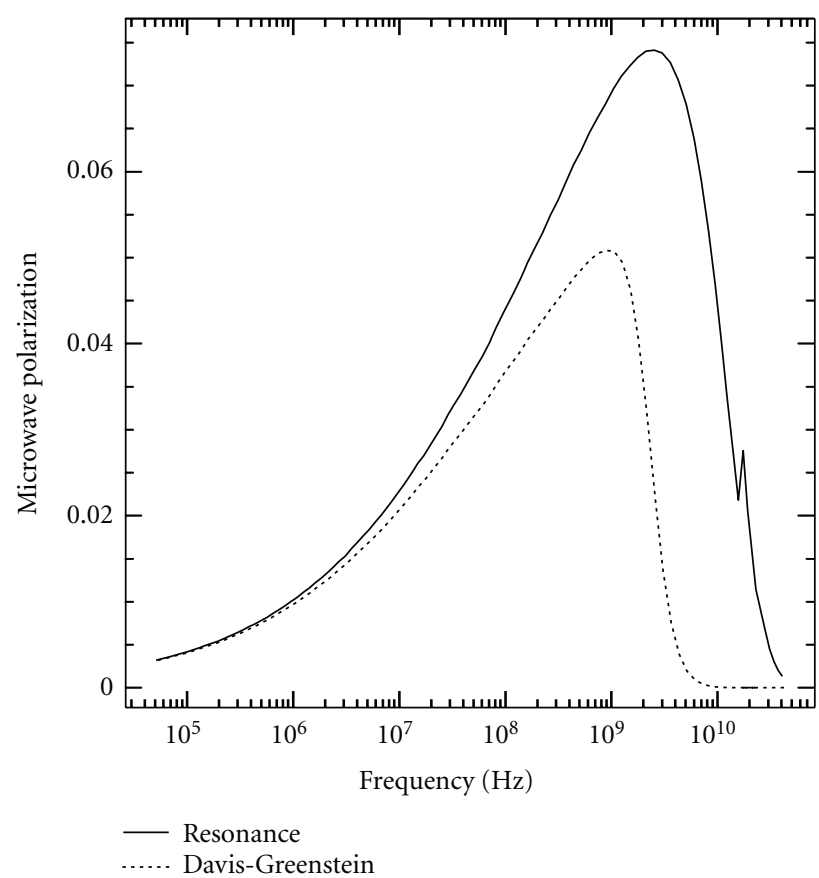

Figure 7: Polarization for both resonance paramagnetic relaxation and Davis-Greenstein relaxation for grains in the cold interstellar medium as a function of frequency (from LD00). For resonance relaxation the saturation effects are neglected, which means that the upper curves correspond to the maximal values allowed by the resonant paramagnetic mechanism.

in relation to its angular momentum (see Lazarian and Roberge [72]). Taking values for Barnett relaxation from Lazarian and Draine [73], we estimate the randomization time of the $10^{-7} \mathrm{~cm}$ grain to be $2 \times 10^{-6} \mathrm{~s}$, which is less than the grain cooling time due to IR emission. As a result, the emanating infrared emission will be polarized very marginally. If, however, Barnett relaxation is suppressed, the randomization time will be determined by inelastic relaxation (Lazarian and Efroimsky [74]) and will be $0.1 \mathrm{~s}$, which would entail a partial polarization of infrared emission.

8.3.2. Can We Constrain the Alignment of Ultrasmall Grains via the Ultraviolet Polarization? PAHs and ultrasmall grains that produce spinning dust emission are likely the same particles that produce the prominent UV absorption feature at $2175 \AA$ (see, e.g., Draine and Li [42]). The lack of polarization excess at $2175 \AA$ is consistent with the expectation that the PAHs are poorly aligned. However, the small degree of polarization (see Wolff et al. [75]) indicates that there must be some residual alignment of ultrasmall grains. The constraint for such a residual alignment can be obtained by fitting the theoretical model with the UV polarization of starlight (Martin [76]). When the residual alignment is available, one can predict the polarization level of spinning dust.

Apart from the emission from spinning dust, another new type of emission from dust is possible. Draine and 
Lazarian [77] noticed that the strongly magnetized material is capable of producing much more microwave thermal emission compared with nonmagnetic grains. They suggested this as a possible alternative to spinning dust emission, which can be responsible for a part or even most of the anomalous microwave emission. Such an emission can be strongly polarized, making anomalous emission an important contaminant in terms of CMB polarization studies.

Further research showed that at the frequencies 20$90 \mathrm{GHz}$, the spinning dust dominates. However, Draine and Hensley [78] performed new calculations of microwave response of strongly magnetic grains. At higher frequencies, this new extensive study of evaluating microwave emissivity of strongly magnetic grains showed that magnetic dipole response of interstellar dust may be extremely important.

\section{Summary}

The principal points discussed previously are as follows.

(i) The model of spinning dust emission proposed by DL98 proved to be capable of explaining anomalous microwave emission, and its predictions were confirmed by numerous observations since the introduction of the model.

(ii) The DL98 spinning dust model has been improved recently by including the effects of thermal fluctuations within dust grains, impulsive excitations with single ions, transient heating by UV photons, triaxiality of grain shape, and compressible turbulence, which made the spinning dust model more realistic.

(iii) Spinning dust emission involves a number of grain physical parameters and environmental parameters. With the latest progress on theoretical modeling and observations, the possibility of using spinning dust as a diagnostic tool for physical parameters of ultrasmall dust is open.

(iv) The spinning dust emission is expected to be partially polarized, but further studies on alignment of ultrasmall grains and modeling of spinning dust polarization are vitally required.

\section{Acknowledgments}

A. Lazarian acknowledges the support of the Center for Magnetic Self-Organization and the NASA Grant no. NNX11AD32G. T. Hoang is grateful for fruitful discussions with Peter Martin on constraining the alignment of ultrasmall grains and polarization of anomalous microwave emission.

\section{References}

[1] F. R. Bouchet, S. Prunet, and S. K. Sethi, "Multifrequency Wiener filtering of cosmic microwave background data with polarization," Monthly Notices of the Royal Astronomical Society, vol. 302, no. 4, pp. 663-676, 1999.
[2] M. Tegmark, D. J. Eisenstein, W. Hu, and A. de OliveiraCosta, "Foregrounds and forecasts for the cosmic microwave background," The Astrophysical Journal, vol. 530, no. 1, pp. 133-165, 2000.

[3] A. Kogut, A. J. Banday, C. L. Bennett, K. M. Górski, G. Hinshaw, and W. T. Reach, "High-latitude galactic emission in the cobe differential microwave radiometer 2 year sky maps," The Astrophysical Journal, vol. 460, no. 1, pp. 1-9, 1996.

[4] A. Kogut, A. J. Banday, C. L. Bennett et al., "Microwave emission at high galactic latitudes in the four-year DMR sky maps," The Astrophysical Journal, vol. 464, no. 1, pp. L5-L9, 1996.

[5] A. De Oliveira-Costa, M. Tegmark, D. P. Finkbeiner et al., "A new spin on galactic dust," The Astrophysical Journal, vol. 567, no. 1, pp. 363-369, 2002.

[6] B. T. Draine and A. Lazarian, "Electric dipole radiation from spinning dust grains," The Astrophysical Journal, vol. 508, no. 1, pp. 157-179, 1998.

[7] B. T. Draine and A. Lazarian, "Diffuse galactic emission from spinning dust grains," The Astrophysical Journal, vol. 494, no. 1, pp. L19-L22, 1998.

[8] W. C. Erickson, "A mechanism of non-thermal radio-noise origin," The Astrophysical Journal, vol. 126, p. 480, 1957.

[9] A. Ferrara and R. J. Dettmar, "Radio-emitting dust in the free electron layer of spiral galaxies: testing the disk/halo interface," The Astrophysical Journal, vol. 427, no. 1, pp. 155-159, 1994.

[10] A. Lazarian and D. Finkbeiner, "Microwave emission from aligned dust," New Astronomy Reviews, vol. 47, no. 11-12, pp. 1107-1116, 2003.

[11] D. P. Finkbeiner, "Microwave interstellar medium emission observed by the Wilkinson Microwave Anisotropy Probe," The Astrophysical Journal, vol. 614, no. 1, pp. 186-193, 2004.

[12] Y. Ali-Haïmoud, C. M. Hirata, and C. Dickinson, "A refined model for spinning dust radiation," Monthly Notices of the Royal Astronomical Society, vol. 395, no. 2, pp. 1055-1078, 2009.

[13] T. Hoang, B. T. Draine, and A. Lazarian, "Improving the model of emission from spinning dust: effects of grain wobbling and transient spin-up," The Astrophysical Journal, vol. 715, no. 2, pp. 1462-1485, 2010.

[14] N. Ysard and L. Verstraete, "The long-wavelength emission of interstellar PAHs: characterizing the spinning dust contribution," Astronomy and Astrophysics, vol. 509, article A12, 2010.

[15] T. Hoang, A. Lazarian, and B. T. Draine, "Spinning dust emission: effects of irregular grain shape, transient heating, and comparison with wilkinson microwave anisotropy probe results," The Astrophysical Journal, vol. 741, p. 87, 2011.

[16] K. Silsbee, Y. Ali-Haïmoud, and C. M. Hirata, "Spinning dust emission: the effect of rotation around a non-principal axis," Monthly Notices of the Royal Astronomical Society, vol. 411, no. 4, pp. 2750-2769, 2011.

[17] B. T. Draine and A. Lazarian, "Microwave emission from galactic dust grains," Microwave Foregrounds, vol. 181, p. 133, 1999.

[18] A. Lazarian and S. Prunet, "Polarized microwave emission from dust," in Proceedings of the Workshop on Astrophysical Polarized Backgrounds, vol. 609, pp. 32-43, March 2002.

[19] A. Li and B. T. Draine, "Infrared emission from interstellar dust. II. The diffuse interstellar medium," The Astrophysical Journal, vol. 554, no. 2, pp. 778-802, 2001.

[20] A. de Oliveira-Costa, A. Kogut, M. J. Deviln, C. Barth Netterfield, L. A. Page, and E. J. Wollack, "Galactic microwave emission at degree angular scales," The Astrophysical Journal, vol. 482, no. 1, pp. L17-L20, 1997. 
[21] E. M. Leitch, A. C. S. Readhead, T. J. Pearson, and S. T. Myers, "An anomalous component of galactic emission," The Astrophysical Journal, vol. 486, no. 1, pp. L23-L26, 1997.

[22] A. De Olivera-Costa, M. Tegmark, L. A. Page, and S. P. Boughn, "Galactic emission at $19 \mathrm{GHz}$," The Astrophysical Journal, vol. 509, no. 1, pp. L9-L12, 1998.

[23] A. de Oliveira-Costa, M. Tegmark, C. M. Gutiérrez et al., "Cross-correlation of tenerife data with galactic templatesevidence for spinning dust?" The Astrophysical Journal, vol. 527, no. 1, pp. L9-L12, 1999.

[24] P. R. McCullough, J. E. Gaustad, W. Rosing, and D. van Buren, "Implications of $\mathrm{H}$ observations for studies of the CMB," Microwave Foregrounds, vol. 181, p. 253, 1999.

[25] A. de Oliveira-Costa, M. Tegmark, M. J. Devlin et al., "Galactic contamination in the qmap experiment," The Astrophysical Journal, vol. 542, no. 1, pp. L5-L8, 2000.

[26] D. P. Finkbeiner, D. J. Schlegel, C. Frank, and C. Heiles, "Tentative detection of electric dipole emission from rapidly rotating dust grains," The Astrophysical Journal, vol. 566, no. 2, pp. 898-904, 2002.

[27] C. L. Bennett, R. S. Hill, G. Hinshaw et al., "First-year Wilkinson Microwave Anisotropy Probe (WMAP) observations: foreground emission," The Astrophysical Journal, vol. 148, no. 1, pp. 97-117, 2003.

[28] D. P. Finkbeiner, M. Davis, and D. J. Schlegel, "Extrapolation of galactic dust emission at 100 microns to cosmic microwave background radiation frequencies using FIRAS," The Astrophysical Journal, vol. 524, no. 2, pp. 867-886, 1999.

[29] G. Langston, A. Minter, L. D’Addario, K. Eberhardt, K. Koski, and J. Zuber, "The first Galactic plane survey at 8.35 and 14.35 GHz," The Astronomical Journal, vol. 119, no. 6, pp. 2801-2827, 2000.

[30] D. P. Finkbeiner, G. I. Langston, and A. H. Minter, "Microwave interstellar medium emission in the green bank galactic plane survey: evidence for spinning dust," The Astrophysical Journal, vol. 617, no. 1, pp. 350-359, 2004.

[31] B. Gold, C. L. Bennett, R. S. Hill et al., "Five-year wilkinson microwave anisotropy probe observations: galactic foreground emission," The Astrophysical Journal, vol. 180, no. 2, pp. 265-282, 2009.

[32] B. Gold, N. Odegard, J. L. Weiland et al., "Seven-year wilkinson microwave anisotropy probe (WMAP $*$ ) observations: galactic foreground emission," The Astrophysical Journal, vol. 192, no. 2, article 15, 2011.

[33] P. Collaboration, P. A. R. Ade, N. Aghanim et al., "Planck early results. Xx. new light on anomalous microwave emission from spinning dust grains," Astronomy and Astrophysics, vol. 536, article A20, 2011.

[34] A. M. M. Scaife, B. Nikolic, D. A. Green et al., "Microwave observations of spinning dust emission in NGC 6946," Monthly Notices of the Royal Astronomical Society, vol. 406, no. 1, pp. L45-L49, 2010.

[35] C. T. Tibbs, R. Paladini, M. Compiègne et al., "A multiwavelength investigation of RCW175: an H II region harboring spinning dust emission," The Astrophysical Journal, vol. 754, no. 2, p. 94, 2012.

[36] S. Casassus, C. Dickinson, K. Cleary et al., "Centimetre-wave continuum radiation from the $\rho$ Ophiuchi molecular cloud," Monthly Notices of the Royal Astronomical Society, vol. 391, no. 3, pp. 1075-1090, 2008.

[37] C. T. Tibbs, N. Flagey, R. Paladini et al., "Spitzer characterization of dust in an anomalous emission region: the Perseus cloud," Monthly Notices of the Royal Astronomical Society, vol. 418, no. 3, pp. 1889-1900, 2011.
[38] C. Bot, N. Ysard, D. Paradis et al., "Submillimeter to centimeter excess emission from the magellanic clouds. II. on the nature of the excess," Astronomy and Astrophysics, vol. 523, p. 20, 2010.

[39] J. C. Weingartner and B. T. Draine, "Radiative torques on interstellar grains. III. Dynamics with thermal relaxation," The Astrophysical Journal, vol. 589, no. 1, pp. 289-318, 2003.

[40] E. M. Purcell, "Suprathermal rotation of interstellar grains," The Astrophysical Journal, vol. 231, pp. 404-416, 1979.

[41] A. Lazarian and W. G. Roberge, "Barnett relaxation in thermally rotating grains," The Astrophysical Journal, vol. 484, no. 1, pp. 230-237, 1997.

[42] B. T. Draine and A. Li, "Infrared emission from interstellar dust. IV. The silicate-graphite-PAH model in the post-Spitzer ERA," The Astrophysical Journal, vol. 657, no. 2, pp. 810-837, 2007.

[43] G. Dobler, B. Draine, and D. P. Finkbeiner, "Constraining spinning dust parameters with the wmap five-year data," The Astrophysical Journal, vol. 699, no. 2, pp. 1374-1388, 2009.

[44] G. Kowal, A. Lazarian, and A. Beresnyak, "Density fluctuations in MHD turbulence: spectra, intermittency, and topology," The Astrophysical Journal, vol. 658, no. 1, pp. 423-445, 2007.

[45] A. Esquivel and A. Lazarian, "Tsallis statistics as a tool for studying interstellar turbulence," The Astrophysical Journal, vol. 710, no. 1, pp. 125-132, 2010.

[46] B. Burkhart, D. Falceta-Gonçalves, G. Kowal, and A. Lazarian, "Density studies of mhd interstellar turbulence: statistical moments, correlations and bispectrum," The Astrophysical Journal, vol. 693, p. 250, 2009.

[47] B. Burkhart, S. Stanimirović, A. Lazarian, and G. Kowal, "Characterizing magnetohydrodynamic turbulence in the small magellanic cloud," The Astrophysical Journal, vol. 708, no. 2, pp. 1204-1220, 2010.

[48] B. T. Draine, "Grain evolution in dark clouds," in Protostars and Planets II, pp. 621-640, University of Arizona Press, Tucson, Ariz, USA, 1985.

[49] H. Yan and A. Lazarian, "Grain acceleration by magnetohydrodynamic turbulence: gyroresonance mechanism," The Astrophysical Journal, vol. 592, no. 1, pp. L33-L36, 2003.

[50] T. Hoang and A. Lazarian, "Acceleration of very small dust grains due to random charge fluctuations," The Astrophysical Journal, vol. 761, no. 2, p. 96, 2012.

[51] T. Hoang, A. Lazarian, and R. Schlickeiser, "Revisiting acceleration of charged grains in magnetohydrodynamic turbulence," The Astrophysical Journal, vol. 747, no. 1, p. 54, 2012.

[52] A. V. Ivlev, A. Lazarian, V. N. Tsytovich, U. de Angelis, T. Hoang, and G. E. Morfill, "Acceleration of small astrophysical grains due to charge fluctuations," The Astrophysical Journal, vol. 723, no. 1, pp. 612-619, 2010.

[53] T. Hoang and A. Lazarian, "Acceleration of small dust grains due to charge fluctuations," arXiv, 1112.3409, 2011.

[54] R. Paladini, G. Umana, M. Veneziani et al., "Spitzer and Herschel multiwavelength characterization of the dust content of evolved HII regions," The Astrophysical Journal, vol. 760, no. 2, 2012.

[55] C. Dickinson, M. Peel, and M. Vidal, "New constraints on the polarization of anomalous microwave emission in nearby molecular clouds," Monthly Notices of the Royal Astronomical Society, vol. 418, no. 1, pp. L35-L39, 2011.

[56] C. H. López-Caraballo, J. A. Rubĩo-Martín, R. Rebolo, and R. Génova-Santos, "Constraints on the polarization of the anomalous microwave emission in the perseus molecular complex from seven-year wmap data," The Astrophysical Journal, vol. 729, no. 1, article 25, 2011. 
[57] N. Macellari, E. Pierpaoli, C. Dickinson, and J. E. Vaillancourt, "Galactic foreground contributions to the 5-year Wilkinson Microwave Anisotropy Probe maps," Monthly Notices of the Royal Astronomical Society, vol. 418, no. 2, pp. 888-905, 2011.

[58] A. Lazarian, "Tracing magnetic fields with aligned grains," Journal of Quantitative Spectroscopy and Radiative Transfer, vol. 106, no. 1-3, pp. 225-256, 2007.

[59] A. Z. Dolginov and I. G. Mytrophanov, "Orientation of cosmic dust grains," Astrophysics and Space Science, vol. 43, no. 2, pp. 291-317, 1976.

[60] B. T. Draine and J. C. Weingartner, "Radiative torques on interstellar grains. I. Superthermal spin-up," The Astrophysical Journal, vol. 470, no. 1, pp. 551-565, 1996.

[61] B. T. Draine and J. C. Weingartner, "Radiative torques on interstellar grains. II. Grain alignment," The Astrophysical Journal, vol. 480, no. 2, pp. 633-646, 1997.

[62] A. Lazarian and T. Hoang, "Radiative torques: analytical model and basic properties," Monthly Notices of the Royal Astronomical Society, vol. 378, no. 3, pp. 910-946, 2007.

[63] A. Lazarian and T. Hoang, "Alignment of dust with magnetic inclusions: radiative torques and superparamagnetic barnett and nuclear relaxation," The Astrophysical Journal, vol. 676, no. 1, pp. L25-L28, 2008.

[64] T. Hoang and A. Lazarian, "Radiative torque alignment: essential physical processes," Monthly Notices of the Royal Astronomical Society, vol. 388, no. 1, pp. 117-143, 2008.

[65] T. Hoang and A. Lazarian, "Grain alignment induced by radiative torques: effects of internal relaxation of energy and complex radiation field," The Astrophysical Journal, vol. 697, no. 2, pp. 1316-1333, 2009.

[66] T. Hoang and A. Lazarian, "Radiative torques alignment in the presence of pinwheel torques," The Astrophysical Journal, vol. 695, pp. 1457-1476, 2009.

[67] D. C. B. Whittet, J. H. Hough, A. Lazarian, and T. Hoang, "The efficiency of grain alignment in dense interstellar clouds: a reassessment of constraints from near-infrared polarization," The Astrophysical Journal, vol. 674, no. 1, pp. 304-315, 2008.

[68] L. Davis and J. L. Greenstein, "The polarization of starlight by aligned dust grains," The Astrophysical Journal, vol. 114, p. 206, 1951.

[69] W. G. Roberge and A. Lazarian, "Davis-Greenstein alignment of oblate spheroidal grains," Monthly Notices of the Royal Astronomical Society, vol. 305, pp. 615-630, 1999.

[70] A. Lazarian and B. T. Draine, "Resonance paramagnetic relaxation and alignmentof amall grains," The Astrophysical Journal, vol. 536, no. 1, pp. L15-L18, 2000.

[71] L. D. Landau and E. M. Lifshitz, Electrodynamics of Continuous Media, Addison-Wesley, Reading, Mass, USA, 1960.

[72] A. Lazarian and W. G. Roberge, "Cosmic rays and grain alignment," Monthly Notices of the Royal Astronomical Society, vol. 287, no. 4, pp. 941-946, 1997.

[73] A. Lazarian and B. T. Draine, "Nuclear spin relaxation within interstellar grains," The Astrophysical Journal, vol. 520, no. 1, pp. L67-L70, 1999.

[74] A. Lazarian and M. Efroimsky, "Inelastic dissipation in a freely rotating body: application to cosmic dust alignment," Monthly Notices of the Royal Astronomical Society, vol. 303, no. 4, pp. 673-684, 1999.

[75] M. J. Wolff, G. C. Clayton, S. H. Kim, P. G. Martin, and C. M. Anderson, "Ultraviolet interstellar linear polarization. III. Features," The Astrophysical Journal, vol. 478, no. 1, pp. 395402, 1997.
[76] P. G. Martin, "On predicting the polarization of low frequency emission by diffuse interstellar terstellar dust," in Sky Polarisation at Far-infrared to Radio Wavelengths: The Galactic Screen Before the Cosmic Microwave Background, M. -A. MivilleDeschênes and F. Boulanger, Eds., vol. 23 of EAS Publications Series, pp. 165-188, 2007.

[77] B. T. Draine and A. Lazarian, "Magnetic dipole microwave emission from dust grains," The Astrophysical Journal, vol. 512, no. 2, pp. 740-754, 1999.

[78] B. T. Draine and B. Hensley, "Magnetic nanoparticles in the interstella medium: emission spectrum and polarization," arXiv, astro-ph.GA, 2012. 

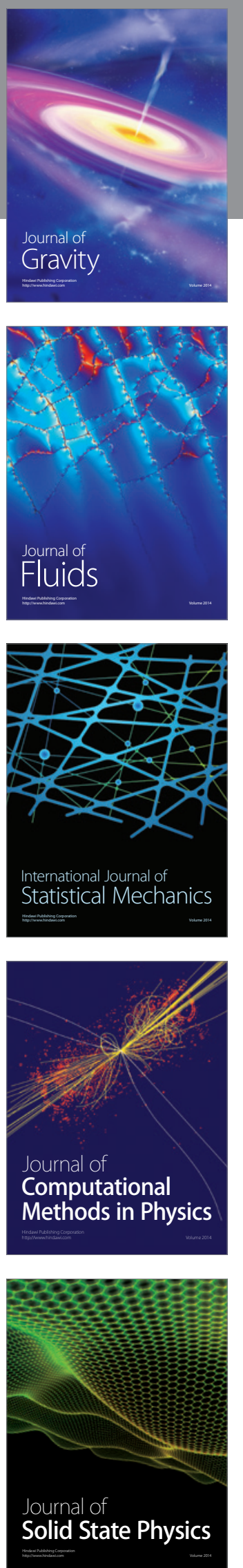

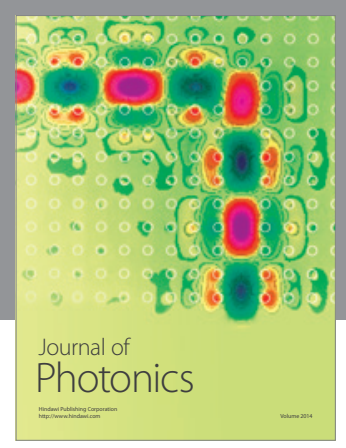

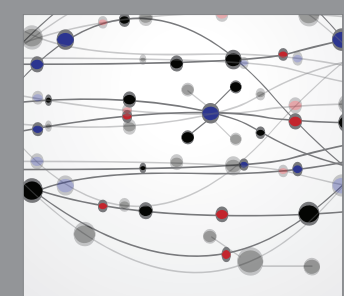

The Scientific World Journal
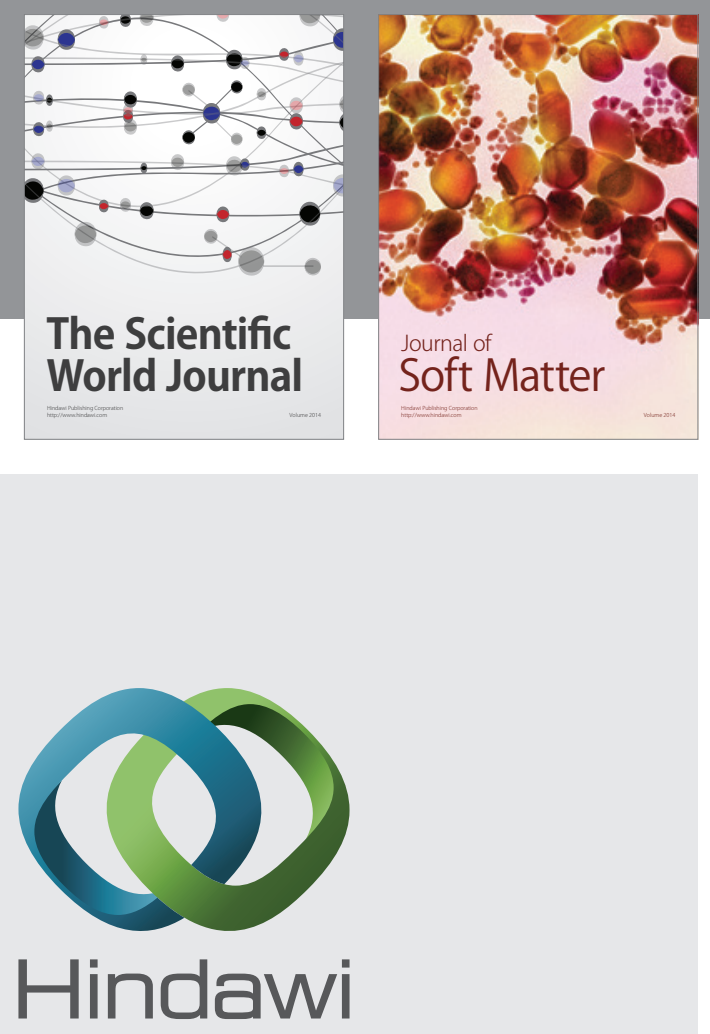

Submit your manuscripts at

http://www.hindawi.com
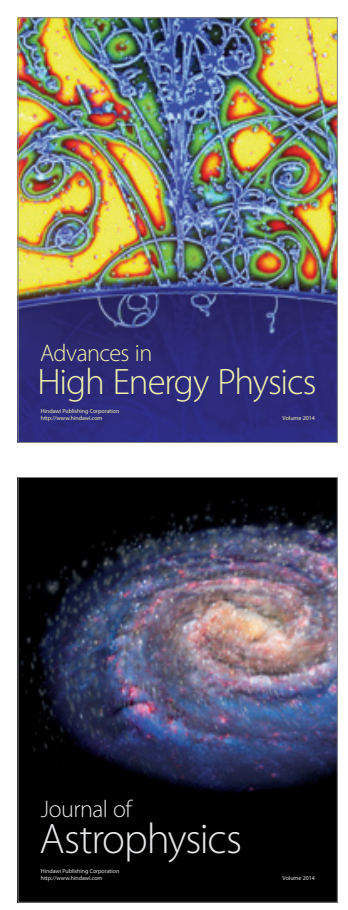
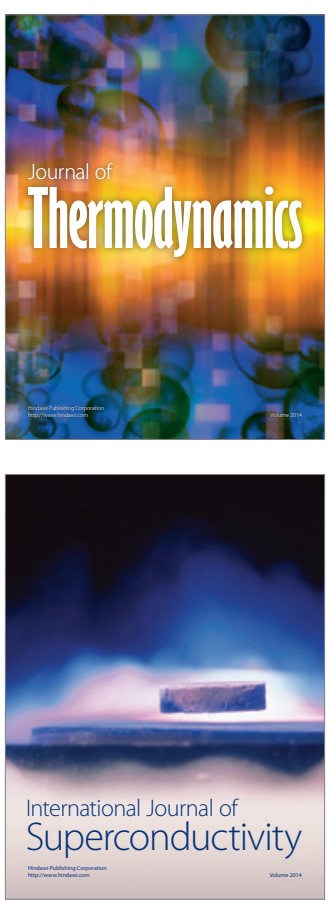
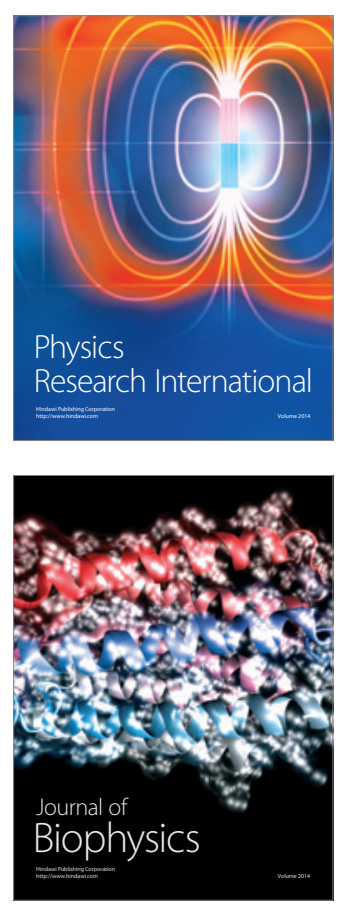
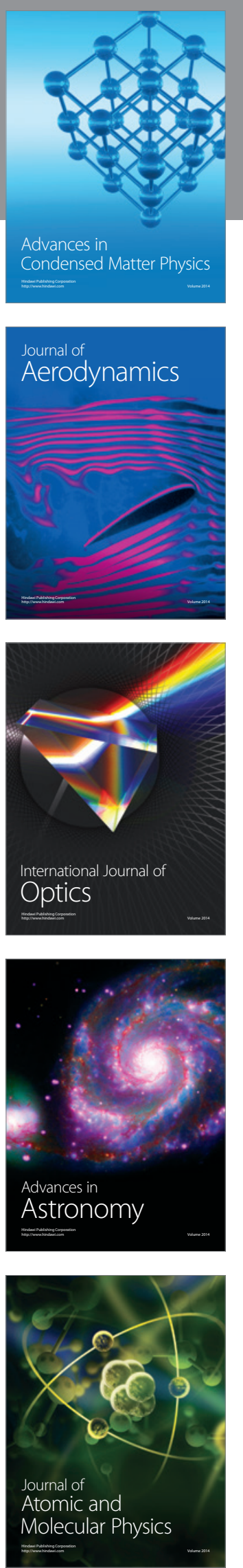